Plumbaum, T., Narr, S., Schwartze, V., Hopfgartner, F., \& Albayrak, S.

\title{
An intelligent health assistant for migrants
}

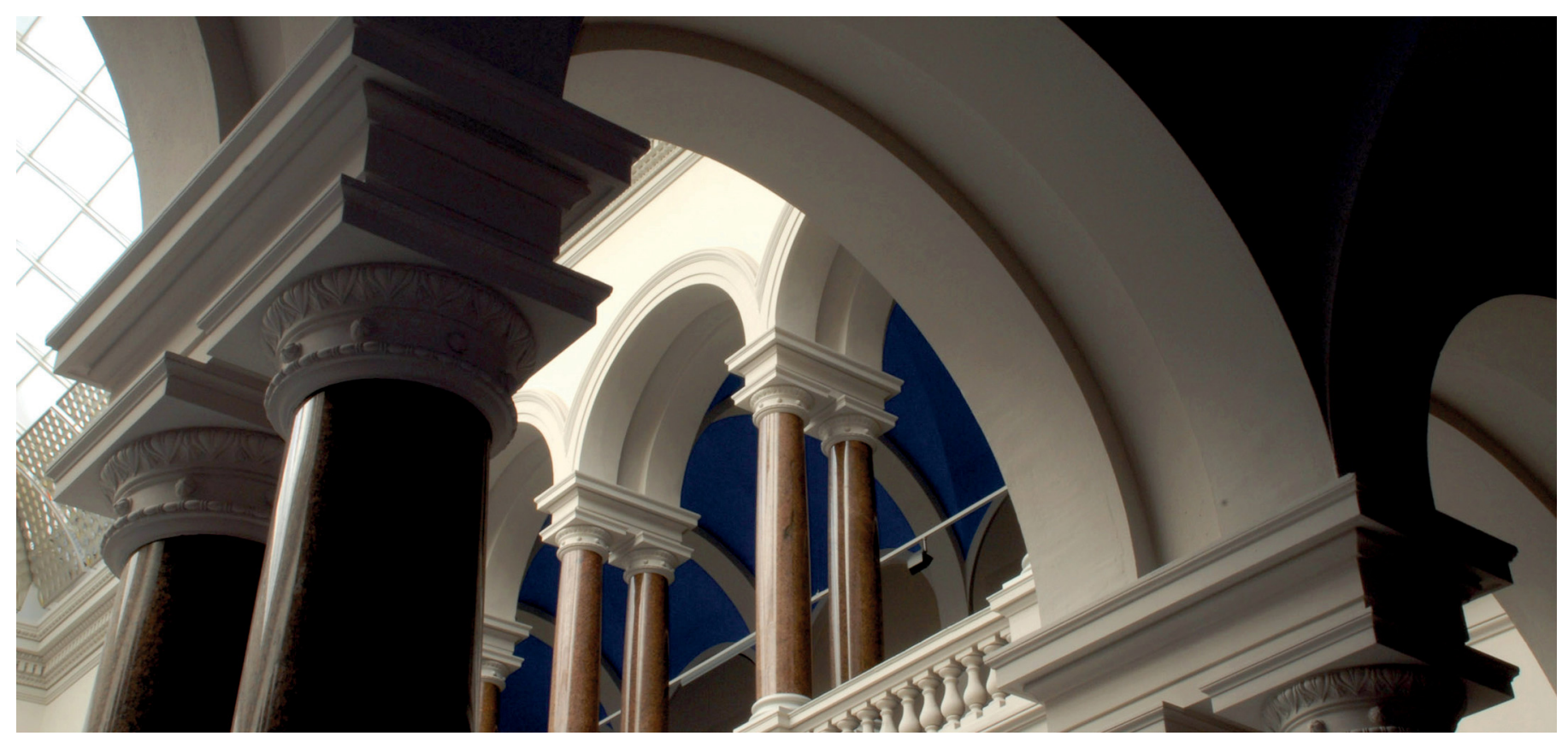

Plumbaum, T., Narr, S., Schwartze, V., Hopfgartner, F., \& Albayrak, S. (2013). An Intelligent Health Assistant for Migrants. In Proceedings of the ICTs for improving Patients Rehabilitation Research Techniques. IEEE. https://doi.org/10.4108/icst.pervasivehealth.2013.252111 


\title{
An Intelligent Health Assistant for Migrants
}

\author{
Till Plumbaum, Sascha Narr, Veit Schwartze, Frank Hopfgartner, Sahin Albayrak \\ Technische Universität Berlin \\ Berlin, Germany \\ \{firstname.lastname\}@dai-labor.de
}

\begin{abstract}
Preventive health is becoming an increasingly important part of our society. In addition to improving people's personal well being, another positive effect is the reduction of costs for medical treatments that put a high burden on individuals and health care providers. Despite these advantages, preventive health services are still not used at a desired level. This is especially true for people with a migration background, since linguistic or cultural barriers prevent them from accessing preventive health services in their host countries. In this paper, we introduce a system which aims to break down these barriers and to give assistance to migrants.
\end{abstract}

\section{INTRODUCTION}

One of the biggest challenges of today's health care providers are the exploding costs for medical treatments of the so-called Western diseases, i.e., diseases such as diabetes or obesity that are due to an unhealthy lifestyle that is common in Western societies. Woolf and Atkins [1] argue that many of these health conditions can be prevented through, for example by educating people about risks of an unhealthy lifestyle. Following the idiom 'prevention is better than a cure', many initiatives have been funded by both governments and health care providers to promote preventive health care. Unfortunately though, information on preventive health care does not reach every member of our society. Although health care providers intend to provide health information services to all their clients, immigrants have been identified as vulnerable population [2] that benefit less from existing health care systems since language and cultural barriers prevent them from using existing prevention services.

In this demo, we present a system that combines a health information service and a prevention service to support migrants in their personal health care. In this paper, we outline the core components of the system that are interconnected to provide an integrated health assistant service.

\section{THE SYSTEM}

The health assistant for migrants consists of two main services that are interconnected, thus providing an integrated preventive health solution. Services include a health information service (HIS) and a prevention service (PS). The HIS improves access to health information. It utilizes a multilingual health ontology, which allows for polyglot queries to retrieve results in the preferred language of the user. The PS supports health care by providing access to nutrition and exercise programs. Both services build on technologies that provide easy access even for users with little technological experience. The services are described in more detail in the remainder of this section.

\section{A. Health Information Service - HIS}

The HIS information service provides a classical search interface that accepts polyglot search queries. Users can enter queries using one or more languages. A user can for instance enter "Fettleibigkeit diyetimi nasıl ayarlamalıyım?" (Translation: "How should I arrange my diet in obesity?"), combining German and Turkish languages, respectively. HIS processes the query and matches the terms of the query to a health ontology. Based on the matched query terms, results are retrieved using a graph-based search method. Search results can be services from health insurances, websites with tips for healthy living or places nearby, e.g. fitness courses in local gyms. Figure 1 shows a screenshot of the HIS GUI where search results to a given query are displayed. The grey box under the search field shows the concepts from the ontology that have been identified in the search query. Underneath it, in the green box, relevant information is displayed. In addition, a list of results for further reading is presented.

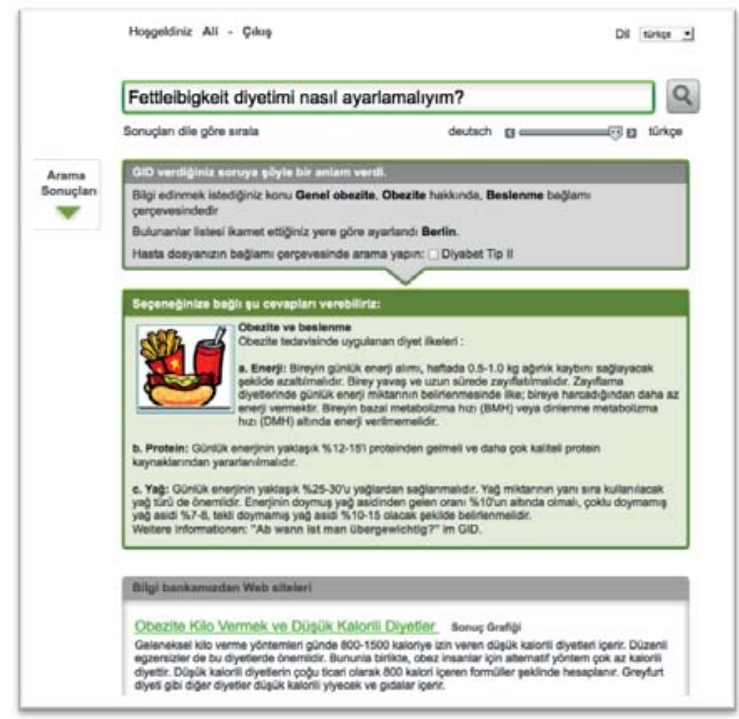

Fig. 1: Results view of the HIS search interface.

For each entry in the result list, users can inspect why this entry is relevant to their given query by clicking on "Result graph" ("Sonuç Grafiği" in above screenshot) which is written next to the title of each entry. A graph is then displayed (see Figure 2) showing identified concepts in the search query and their relationship to other concepts as defined in the ontology.

By supporting multi-lingual querying and retrieval, HIS especially helps people to bridge language barriers. In combination with the prevention service presented below, the 


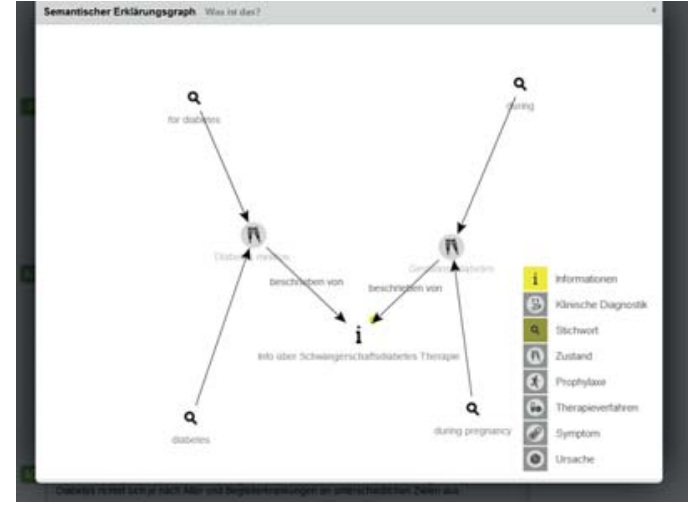

Fig. 2: Graph Search Explanation View showing the path from the search query to the retrieved result.

HIS service can therefore be an important building block for promoting healthy living to all members of our society.

\section{B. Prevention Service - PS}

The prevention service, PS, consists of two subsystems focusing on nutrition and activity support for people. Figure 3 shows the start screen of the PS. On the left hand side of the GUI is a calendar view that lists appointments (e.g., medical treatments, gym classes) that a user should be aware of. On the right hand side of the GUI, the user has access to a nutrition assistant and an activity assistant that form part of the PS. Both services are described in the remainder of this section.

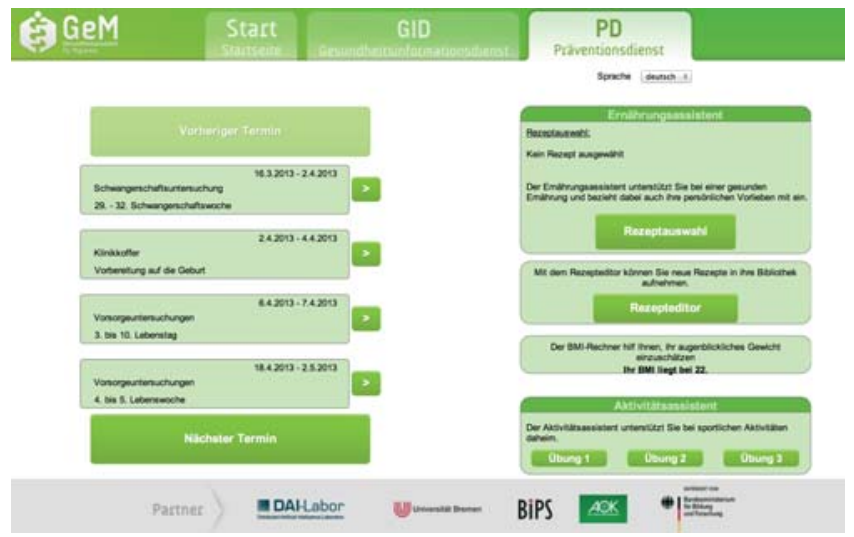

Fig. 3: Calender view (left) and access panels to the nutrition and activity assistant (right) of the prevention service.

1) Nutrition Assistant: The nutrition assistant helps user eat in a healthy way. Users can get a list of cooking recipes that are tailored to their physical activity, medical conditions and cultural background. For example, if the user has diabetes, recipes with high sugar content are avoided. Besides, the interface provides an overview on how healthy their current lifestyle is with respect to physical activities and nutrition habits. This helps users to learn about the food they eat. After selecting a specific dish, the user gets a list of ingredients and a step-by-step instruction on how to cook the meal.
2) Activity Assistant: The activity assistant intends to defeat one's weaker self. By using game mechanics and rewards, people are motivated to do physical exercises at home [3]. As shown in Figure 4, users see a digital trainer who demonstrates an exercise (e.g., Jumping Jacks) that should be imitated by them. Users' motions are tracked using a XBOX Kinect 3D camera and compared to the instructed movements. For each activity, the users can earn activity points. The more exercises the users perform, the more activity points they earn. These points are directly fed into the nutrition assistant, i.e., the food suggestion depends on the users' individual energy expenditure.

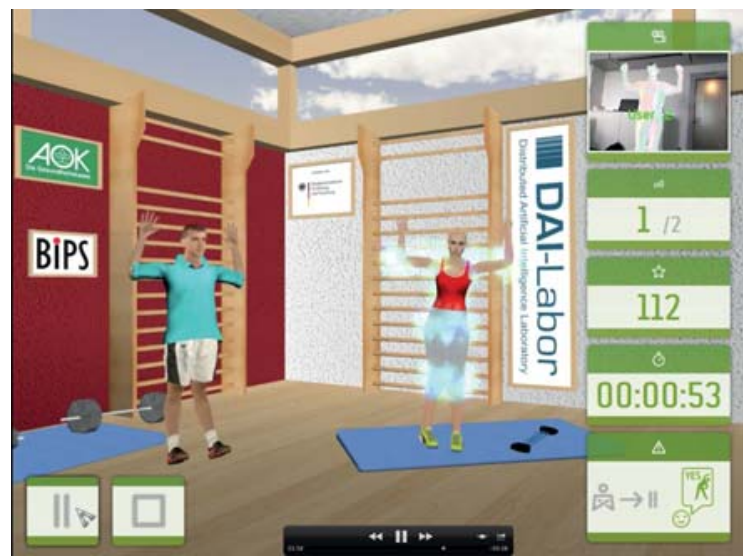

Fig. 4: Activity Assistant: The user, shown in the upper right corner, is captured by the XBOX Kinect 3D camera and should follow the exercises of the trainer (left).

\section{Demo System}

The health assistant system, consisting of the HIS and PS services, integrates various services to help migrants live a healthier life. Given the objective of the research project, the system has a strong focus on helping migrants to overcome the initial language barrier by providing them multi-lingual tips for a healthy lifestyle, information about health care services in their host country and managing their daily nutrition intake and physical exercises. The system was evaluated in a user study with 12 Turkish families in a mid-size town in Germany. Results were all positive and resulting in efforts of a big German health insurance company to implement such system for their members. In this demo, we present a fully working prototype. Users can search for health information in German, Turkish and partially English languages. As prove of concept, the HIS service currently provides information about diabetes, pregnancy and obesity. The nutrition service is fully functional and can be showcased at the conference. Due to constructional restrictions with the $\mathrm{XBOX}$ Kinect $3 \mathrm{D}$ camera, the activity assistant can be presented as a video only.

\section{REFERENCES}

[1] S. Woolf and D. Atkins, "The evolving role of prevention in health care," American Journal of Preventive Medicine, pp. 13-20, 042001.

[2] K. Derose, J. Escarce, and N. Lurie, "Immigrants and health care: Sources of vulnerability," Health Affairs, pp. 1258-1268, 092005.

[3] M. N. K. Boulos, "Xbox 360 kinect exergames for health," Games for Health Journal, vol. 1, pp. 326-330, 2012. 